\title{
La liaison en français contemporain: normes, usages, acquisitions*
}

\author{
JULIEN EYCHENNE ${ }^{a}$ et BERNARD LAKS \\ ${ }^{a}$ Hankuk University of Foreign Studies \\ ${ }^{b}$ Institut Universitaire de France et Université Paris Ouest
}

\section{LA LIAISON}

La phonologie du français présente un phénomène bien connu de sandhi externe à la jointure de mot appelé liaison : devant une unité lexicale à initiale vocalique, un mot se terminant apparemment par une voyelle s'enchaîne syllabiquement en

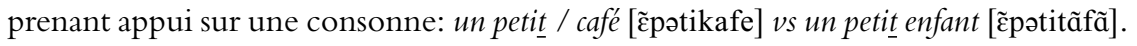
Cette consonne de liaison (CL), ici le /t/, qui n'apparaît que lorsque le mot de droite commence par voyelle, a été analysée comme latente, épenthétique ou supplétive, appartenant au mot de gauche (mot I, MI ci-après), de droite (mot 2, M2 ci-après) ou à aucun selon les auteurs, nous y reviendrons. La liaison est un phénomène complexe dont la phénoménologie est encore aujourd'hui sujette à recherches et à débats. Dans la littérature classique, orthoépique ou descriptive, comme dans les recherches les plus actuelles, la liaison est considérée comme un phénomène multi-paramétrique et tous les niveaux linguistiques sont convoqués : phonologie, prosodie et syllabation, morphologie, syntaxe, lexique et sémantique, diachronie, orthographe et différentiation des styles ; et on doit encore y ajouter la sociolinguistique et la fragmentation sociale des communautés linguistiques (cf. Laks 2005). Tous les niveaux d'analyse grammaticale interne sont ainsi impliqués, de même que sont concernés toutes les dimensions de la variation externe : variation dans le temps, dans l'espace géographique et dans l'espace social, variation dans l'espace stylistique des genres de discours. Enfin, s'ajoute à ces dimensions internes et externes de variation, une variation proprement inhérente (Labov I994), irréductible à toutes les autres. Les analyses classiques, qu'elles soient à visée didactique, orthoépique ou normative divergent assez profondément sur la phénoménologie mais s'accordent sur le processus. Les analyses formelles de type génératif ou postgénératif reprennent sans la critiquer ou l'élargir la phénoménologie la plus reçue et convergent sur la mécanique procédurale. Dans la dernière période au contraire, tant la description fine du processus et sa variation multi-paramétrique que le processus de liaison lui-même ont fait l'objet de réanalyses profondes qui modifient très sensiblement la compréhension que l'on peut avoir aujourd'hui de la liaison (voir les contributions dans Soum et al. 20I4). L'objectif de ce numéro spécial est d'illustrer quelques-unes de ces pistes d'analyse les plus récentes.

* La contribution de Julien Eychenne à cet article a été soutenue par le Hankuk University of Foreign Studies Research Fund 2016 
Dans son acception traditionnelle, la liaison consiste à " prononcer devant un mot commençant par voyelle une consonne finale, muette en dehors de cette condition " (Fouché I959: 434), comme on peut le voir dans les exemples en (I) :

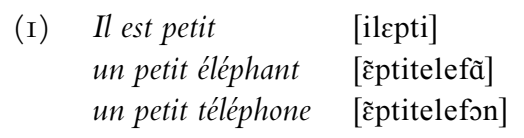

Les descriptions traditionnelles et les manuels orthoépiques distinguent par ailleurs trois grandes catégories de liaison : obligatoire, facultative, interdite (Delattre I966), selon que la CL est réalisée de manière catégorique (petit $[\mathrm{t}]$ ami), variable (elle est [t] arrivée ou elle est / arrivée) ou ne peut normalement être réalisée (l'enfant / a froid). Delattre, après Grammont (I9I4), met de plus en avant un principe général, que l'on appellera ici principe de cohésion (2), selon lequel la réalisation de la CL est conditionnée par le degré d'affinité syntactico-sémantique entre le MI et M2.

(2) Principe de cohésion

"La liaison se fait dans la mesure où l'usage a consacré l'extrême étroitesse d'union de deux mots ou classes de mots. » (Delattre I966 : 39)

Par ailleurs, l'approche classique accorde une place importante à l'information proprement lexicale ou morpho-lexicale dans la mesure où elle relève de très nombreux exemples de constructions figées ayant chacune un comportement idiosyncratique, avec ou sans liaison : pot $[\mathrm{t}]$ à lait mais pot / à tabac, un long $[\mathrm{g}]$ été mais de long / en large, des vers [z] illustres mais un vers / atone, de temps [z] en temps mais le temps / était chaud, un pied [t] à terre, mais mettre le pied / à terre, etc. Ainsi, si dans la perspective classique la liaison est susceptible d'un classement typologique tripartite (Delattre I95 I ; Fouché 1959), elle ne saurait être ramenée à un mécanisme unique et simple de prononciation devant un $\mathrm{M} 2$ à initiale vocalique d'une consonne graphique muette ailleurs.

En effet, si la notion de consonne muette correspond grosso modo à une consonne finale de mot graphique non prononcée, ce n'est pourtant pas toujours le cas. La question de la liaison recoupe ainsi la question plus large des consonnes latentes du français (Laks 2005; Bonami, Boyé et Tseng 2005), qui peuvent faire surface dans la morphologie dérivationnelle (port [por] $\rightarrow$ portuaire [portyer]) et flexionnelle, en particulier dans les alternances de genre (petit [pati] $\rightarrow$ petite [potit]) et de nombre (petits amis [pətizami]). La consonne de liaison peut ainsi être identique à la consonne que l'on trouve en dérivation (petit [pəti]; petite [pətit] ; petit ami [pətitami]) ou être différente (grand [grã] ; grande [grãd] ; grand ami [grãtami]). Enfin, la consonne qui apparait en dérivation n'est pas toujours constante (vert [ver] ; verte [vert] ; verdir [verdir]), et les consonnes latentes ne sont pas toujours marquées dans la graphie : four $\sim$ fournée, jour $\sim$ journée $e^{1}$.

${ }^{1}$ On notera de ce point de vue que l'orthographe française a beaucoup fluctué, et ce de manière assez arbitraire : si l'on trouve en ancien français les graphies forn et jorn pour 
Les descriptions classiques et la conception orthoépique sont donc loin d'être satisfaisantes : la graphie n'est pas un guide infaillible et la notion de consonne latente est loin d'aller de soi. De plus, l'analyse descriptive, le plus souvent normative et simplement reprise des travaux antérieurs, ne s'appuie pas sur une analyse phénoménologique précise des usages attestés dans leur diversité et leur variabilité multi-paramétrique interne comme externe. Enfin, la problématique de la liaison saisie dans l'ensemble de ses dimensions induit de redoutables questions quant à l'apprentissage, natif ou non natif, des mécanismes, des comportements et des usages, mais aussi au plan cognitif et mémoriel, des stockages et des représentations.

\section{LES APPROCHES FORMELLES PHONOLOGIQUES}

Dans l'histoire des descriptions et des analyses de la liaison, la période générative et post-générative représente un moment particulier. Dès I965, Schane, par ailleurs fin connaisseur des traditions descriptives du français, avait proposé une réduction de la liaison à sa seule dimension morphophonologique (Schane 1965, I967). S'appuyant sur une bibliographie sommaire de moins de 25 entrées consacrées au français et sur seulement 75 exemples repris des grammaires normatives (Grevisse 1936), il propose un système formel pour analyser tout l'ensemble des phénomènes de la phonologie du français, dont la liaison consonantique et l'élision vocalique $^{2}$, et d'une bonne partie de sa morphologie. Son objectif, typique de l'entreprise générative, ne consiste ni à ressourcer les descriptions de la liaison ni à en améliorer l'analyse mais à construire pour ces 75 exemples un système de pas moins de 4I règles ordonnées qui permettent formellement de dériver toutes les liaisons, les élisions et les troncations du français (cf. Goldsmith et Laks $2000: 3)$.

Comme cela a souvent été souligné, pour aboutir à ce nouveau schéma formel, Schane confond sciemment un processus vocalique, l'élision, qui voit la disparition à l'oral et à l'écrit d'une voyelle graphique, et un processus consonantique, qui voit à l'oral seulement une consonne graphique ne pas être prononcée devant initiale consonantique. Or ce parallélisme n'est que de façade et, si souvent noté avant lui par les phonologues du français, il n'avait pas prêté à d'autres conséquences précédemment, c'est que dans les deux processus, toutes les contraintes contextuelles et les conditionnements morphologiques different si profondément qu'il est impossible de tenir élision et liaison pour les deux résultats d'un même processus abstrait. Critiquée au sein même du paradigme génératif dès son apparition pour son inadéquation empirique (Dell I973) et son absence de

four et jour, dont le /n/ final n'était déjà plus prononcé mais était toujours présent dans les dérivés, on assiste également à la réintroduction de lettres muettes finales dont le seul but est de rappeler l'étymologie latine (cf. le moyen français cor, devenu corps en français contemporain sur la base du latin corpus).

2 L'élision vocalique consiste à syncoper une voyelle devant voyelle : le palais [ləpale] vs l'hôtel [lotsl] 
prise en compte des contraintes morphosyntaxiques (Milner 1967; Selkirk 1972), ce traitement sera rapidement abandonné, et Schane le désavouera d'ailleurs luimême (Schane 1974). Mais sa proposition formelle aura une influence profonde et durable sur les analyses de la liaison. En effet, Schane introduit subrepticement l'idée que la question centrale concernant la liaison est celle de son processus de mise en œuvre, et accessoirement de sa formalisation. La variation des usages, les conditionnements contextuels de tous niveaux qui la contraignent sont relégués à la marge d'une linguistique descriptive et peuvent être ignorés au profit de son traitement strictement procédural. Or pour l'immense majorité des linguistes et phonologues du français depuis le I6ème siècle c'est exactement l'inverse qui est vrai! La liaison en tant que processus est totalement claire et parfaitement comprise depuis toujours. S'attacher, comme le font toutes les approches génératives et post-génératives jusqu'à Encrevé (I988) et encore récemment Scheer (20I5) et Scheer et al. (20I5) qui le reprennent, à formaliser ce mécanisme n'apporte guère d'information linguistique supplémentaire par rapport à la définition en langage ordinaire que nous avons rappelée plus haut. Ce qui importe, ce n'est pas tant de formaliser le processus de syllabation d'une CL sur un $\mathrm{M} 2$ à initiale vocalique, mais de comprendre ce que peut être une consonne «muette» et dans quelles conditions précises elle peut ou non se prononcer.

Considérons ce processus de liaison tel qu'il est formalisé dans un cadre autosegmental post-génératif par Scheer (2015 : IOO). (3a) présente la structure proposée dans ce cadre pour un mot comme petit qui présente une forme sans $/ \mathrm{t} /$ devant initiale consonantique (petit café [patikafe]) et avec / $\mathrm{t} /$ devant initiale vocalique (petit enfant [ع̃patitãfã]). ( $3 \mathrm{~b}$ ) présente la structure d'un mot comme pépite dont la dernière syllabe est fermée et qui apparaît avec un /t/ devant initiale vocalique comme devant initiale consonantique. Le français connait un processus très général d'enchaînement qui lie dynamiquement les unités lexicales les unes aux autres au sein d'unités prosodiques plus larges appelées syntagmes phonologiques. Ce processus bien connu d'enchaînement prend la forme de la resyllabation d'une consonne finale de mot sur l'initiale du mot suivant si elle est vocalique (Delattre I95I ; Fouché I959; Grammont I9I4). Ce processus d'enchaînement est illustré en (4) où la consonne finale de pépite est resyllabée à l'initiale de ovale : son liage avec la syllabe initiale de ovale entraîne son déliage de la syllabe finale de pépite. Comme le souligne fort justement Encrevé (I988), la liaison en français n'est qu'un cas particulier de l'enchaînement, c'est-à-dire précisément de cette resyllabation des consonnes finales sur les initiales vocaliques que nous venons d'illustrer. Mais si la liaison est un cas particulier, ce qui importe alors de comprendre, d'analyser et éventuellement de formaliser, c'est précisément ce qui en fait un cas particulier. Ce ne peut être l'enchaînement puisque comme nous venons de le voir, le processus d'enchainement est strictement identique dans un cas d'enchaînement d'une consonne finale fixe comme dans (4) et d'une consonne de liaison (CL) comme dans ( $5 \mathrm{a})$. La seule différence tient à la représentation de la CL dans ( $5 \mathrm{a})$. $\mathrm{Le} / \mathrm{t} / \mathrm{de}$ petit n'étant pas lié au squelette, il n'a pas besoin d'être dissocié, comme il doit l'être dans pépite. 
(3) Consonne de liaison flottante vs consonne finale fixe

(3a)

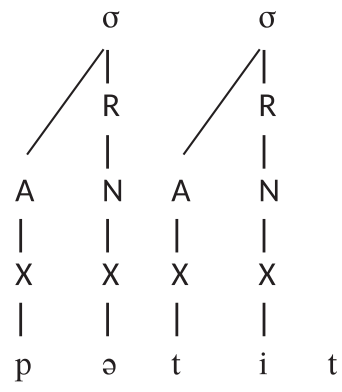

(3b)

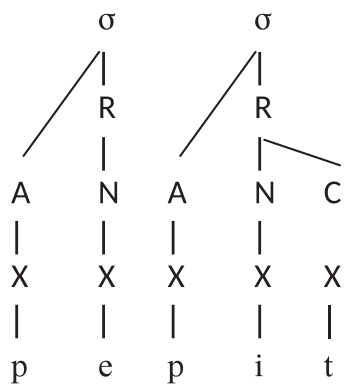

(4) Resyllabation d'une consonne fixe
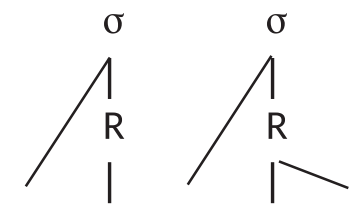

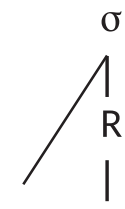

A N

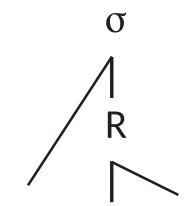

A N C

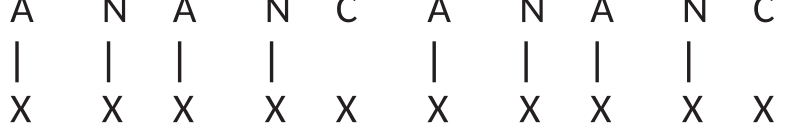<smiles>CC1C=CC1[AlH]</smiles>

$p$ e p i t
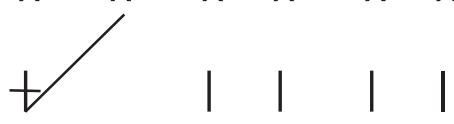

o $\quad$ V $\quad$ a 1

Dans [pətikafe] ( $5 \mathrm{~b})$, l'enchainement ne peut avoir lieu car l'association du /t/ à l'initiale est impossible puisque que cette dernière est déjà liée à une autre consonne. /t/ reste donc dissocié, c'est-à-dire non syllabé et non réalisé.

(5) Réalisation vs non-réalisation d'une consonne flottante

(5a)
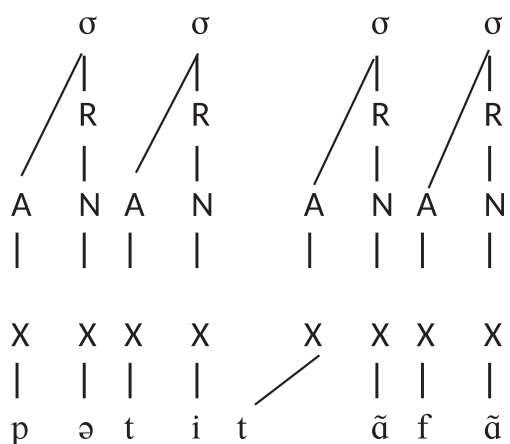

(5b)
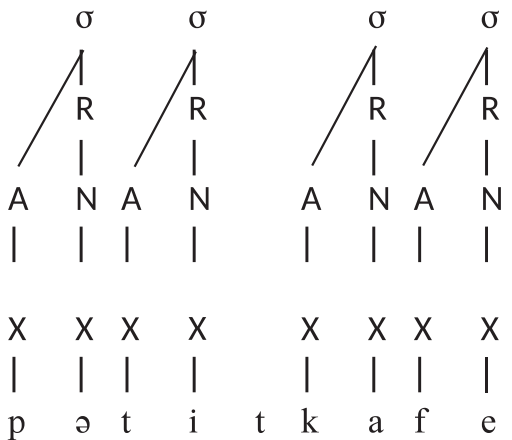
Comme on le voit, du point de vue des mécanismes, quelle que soit la manière de les envisager (processus, règles, contraintes), il n'y a donc rien de spécifique ou de particulier dans le cas de la liaison. C'est l'enchaînement qui est à l'œuvre, sans aucune autre spécification. C'est une illusion très résistante introduite par le traitement génératif initial de Schane que de penser qu'il y aurait en français un mécanisme de liaison ou plus exactement de non-liaison. En effet, dans le traitement de Scheer et d'Encrevé, la CL est toujours présente dans l'entrée lexicale de Mr. Dans le cas normal, sans autre stipulation, elle est resyllabée sur M2 s'il présente une initiale vocalique. Ce n'est que lorsque cette syllabation est bloquée par la présence d'une initiale consonantique (ou d'un $h$ aspiré) dans M2 que l'enchaînement n'a pas lieu. On en déduit donc qu'il y a toujours liaison : dès que l'enchaînement CV est possible, il a lieu (cf. Tranel I995).

Or ceci est manifestement faux. Comparons par exemple nous sommes des [z] amis où la liaison est catégorique et nous ne sommes pas / amis où la liaison est variable et peut ne pas avoir lieu. Dans les deux cas, les segments impliqués et les structures syllabiques sont identiques, mais dans des [z] amis omettre la resyllabation du $<s>$ du déterminant sur le /a/ initial est impossible : la liaison du déterminant pluriel est normalement réalisée de manière catégorique, et ce quel que soit le style. Par contre, ne pas réaliser la liaison de la négation pas est parfaitement possible et, de fait, dans la plupart des styles, cette liaison est souvent absente avec le $<s>$ du pas de négation. Analyser et expliquer la liaison en français, pour autant que les notions d'adéquation observationnelle, descriptive et explicative dont on se revendique dans le cadre génératif et post-génératif aient un sens, ce n'est pas, on le voit, décrire le processus syllabique de l'enchainement, c'est analyser les raisons pour lesquelles, dans le même environnement segmental et syllabique, la liaison est selon les cas catégorique, variable ou absente.

La ligne argumentative que nous venons de rappeler est bien connue. Pour la période récente, elle a été exposé avec beaucoup de détails par Morin (Morin I986; Morin 2003 ; Morin 2005a ; Morin 2005b ; Morin et Kaye 1982) : les contraintes qui pèsent sur la liaison en français sont d'ordre morphosyntaxique, syntaxique ou même sémantique. Elles doivent être spécifiées type de construction par type de construction (cf. aussi Bybee 200Ia,b). Le bilan critique des analyses formelles que nous venons de voir est donc assez simple à résumer : il n'y a pas à strictement parler de processus phonologique de liaison en français. Au plan phonologique, c'est le processus général d'enchaînement et de resyllabation actif partout ailleurs dans la langue qui s'applique. Toute la phénoménologie de la liaison tient alors au marquage lexical particulier de certaines consonnes et donc l'analyse de la liaison doit porter sur le statut lexical de cette CL et sur les conditions constructionnelles spécifiques qui contraignent la variabilité de sa réalisation ou de sa non-réalisation. Comme nous l'avons rappelé plus haut, c'est exactement la position qui avait été défendue par tous les linguistes du français, jusqu'à la phonologie générative et post-générative. C'est également la position qui a été reprise plus récemment par un grand nombre de linguistes qui ont cherché à analyser les patrons de variabilité et les contraintes multi-paramétriques de tous niveaux qui pèsent sur la liaison en se fondant sur la 
linguistique de corpus et sur une analyse précise des usages tels qu'ils sont attestés (Côté 20I I ; Durand et al. 20I I ; Laks et Calderone 20I3).

\section{STATUT DE LA CL ET STRUCTURE DE L'INFORMATION LEXICALE}

Dans l'histoire des traitements de la liaison, trois types d'analyses ont été proposées pour rendre compte du statut des CL. Ces trois types d'analyse correspondent toutes à un marquage lexical spécifique, mais il y a quelques différences. La CL peut être analysée comme latente, comme épenthétique ou les formes peuvent être considérées comme supplétives (voir Bonami et Boyé 2003 ; Bonami et al. 2005 ; Côté 2005, 20 I I, pour de nombreuses références). L'analyse supplétive se fonde sur le fait qu'un certain nombre d'adjectifs, de pronoms, de déterminants présentent deux formes lorsqu'ils sont préposés à un nom, selon que celui-ci commence par une voyelle ou une consonne. On a ce panneau [səpano] mais cet anneau [sctano], un beau camarade [bokamarad] mais un bel ami [belami], un vieux camarade [vjøkamarad] mais un vieil ami [vjejami], un mauvais compromis [movekõpromi] mais un mauvais argument [movezargymã], etc. L'analyse supplétive postule que les entrées lexicales correspondantes présentent deux formes, une forme dite longue et une forme dite courte. La question de savoir si la forme longue correspond ou non à la forme féminine a été beaucoup discutée depuis la célèbre analyse de Marguerite Durand (Durand I936 ; Pichon I938). Elle ne nous concernera pas ici. Nous retenons que l'analyse supplétive présente un certain nombre d'arguments, pas tous liés à la liaison, pour défendre la nécessité d'inscrire deux formes morphologiquement différentes dans un certain nombre d'entrées lexicales. L'information lexicale doit alors indiquer quelle forme apparait devant $M_{2}$ à initiale vocalique, donc quelle forme apparait en liaison. Dans cette approche donc ce n'est pas la consonne de liaison CL qui est lexicalement marquée mais la forme supplétive elle-même qui doit l'être car l'alternance est plus complexe que la simple mise en œuvre d'une consonne muette.

La deuxième analyse concernant la consonne de liaison lui donne le statut de consonne latente, à savoir une consonne qui est grammaticalement présente mais qui peut ne pas se prononcer (Pichon I938: 26). Les analyses génératives et post-génératives depuis Schane ont, comme nous l'avons vu, systématiquement repris cette position en notant la CL dans la forme lexicale et en tentant de formaliser les conditions suivant lesquelles elle n'apparait pas en surface. L'analyse par latence pose la question de la motivation de la consonne concernée. La consonne latente a souvent été motivée par la forme orthographique du mot. Les consonnes orthographiques sont celles qui sont stockées dans ce que Martinon (I9I $3: 356$ ) appelait avec ironie le « conservatoire ou musée des liaisons » et il y a une correspondance stricte en français métropolitain où toute CL est nécessairement une consonne graphique. Or, premièrement, l'inverse n'est pas vrai et toute consonne graphique ne peut pas faire CL : le $<t>$ de $e t$, le $\langle p>$ de champ, le $<s>$ de univers etc. ne lient jamais. Mais surtout, fonder la latence sur la graphie implique une thèse concernant la relation entre code oral et code écrit extrêmement discutable 
(cf. Laks 2005) que Pichon comme son co-auteur Damourette rejetaient radicalement en s'opposant à ce qu'ils appelaient avec un certain mépris "l'orthographisme primaire» (Damourette et Pichon I9I I-I927). Pour Pichon la consonne latente ne peut être motivée que du point de vue interne. Elle implique ce qu'avec Damourette il appelle des muances : une unité lexicale peut se présenter selon plusieurs formes et l'alternance porte sur cette fameuse consonne latente. On voit que du point de vue de la quantité et de la qualité de l'information lexicale on est exactement avec cette conception de la latence dans le cas de la supplétion. Comme dans le cas de la supplétion, on ne peut même pas s'appuyer sur le radical dérivationnel car quelques consonnes paradigmatiquement motivées ne font pourtant jamais liaison. Comparons petit, petitesse, petitement, petit $[\mathrm{t}]$ ami avec temps, temporaire, temporel, temps / épouvantable, ou encore camp, campement, camper et camp / étroit. Marquer la consonne latente comme CL peut donc être vu comme une simple variante notationnelle de l'analyse supplétive.

La troisième solution consiste à analyser la CL comme épenthétique (Côté 20II, 2014 ; Tranel I98I, 2000). De fait dans certaines variétés du français, notamment au Canada on relève des consonnes purement épenthétiques qui ne sont ni graphiques, ni paradigmatiques, ni normatives. Traiter la CL comme une consonne épenthétique implique de stipuler sur quel mot s'opère l'épenthèse. Côté présente en détail les arguments qui conduisent à rattacher la consonne à MI, ou à $\mathrm{M}$ 2. Lorsque le matériel consonantique correspond à un morphème de nombre petits $[\mathrm{z}]$ amis ou de personne nous $[\mathrm{z}]$ avons, on sait depuis Hindret (I687) que l'analyse la plus cohérente consiste à dire que le nombre se marque en début de mot lorsqu'il commence par une voyelle et donc à attacher cette marque à l'initiale de M2. Même si cette analyse est contradictoire avec la forme graphique, Gougenheim (1938), Morin (1986) et Morin et Kaye (1982) ont présenté des arguments très solides pour l'appuyer (voir également Côté 20 I I). La question du rattachement ne nous occupera pas directement ici. En effet, si la CL est rattachée au MI, on se trouve du point de vue de la structure informationnelle dans le cas de la supplétion : les déterminants et pronoms ont deux formes, courte devant consonne, longue devant voyelle. Mais pour ces catégories morphologiques, la liaison, et donc l'enchaînement, sont obligatoires, stables et ne souffrent aucune exception. On a donc des constructions stabilisées et stockées comme telles. Si la CL est au contraire rattachée à $\mathrm{M}_{2}$, cela ne change rien dans la mesure où on a toujours une liaison obligatoire enchaînée et où donc la construction est lexicalement représentée. En d'autres termes, les proclitiques forment une construction soudée avec la catégorie principale sur laquelle ils s'appuient, et des [z] amis ou nous [z] allons constituent un seul « mot».

Ce tour d'horizon montre que la liaison ne peut être envisagée comme un phénomène unitaire et strictement phonologique ; il s'agit d'un phénomène multifactoriel dont une partie non négligeable relève du stockage lexical. Comprendre la liaison dans toute sa complexité requiert donc d'asseoir la description et la modélisation sur des données attestées, qu'elles soient expérimentales ou qu'elles proviennent de corpus de parole (Barreca 2015 ; Côté 20 I i ; Durand et Lyche 2008 ; 
Durand et al. 20 I I). C'est dans cette optique de ressourcement empirique qu'a été conçu le présent volume.

\section{PRÉSENTATION DU NUMÉRO}

Si les chercheurs réunis dans ce volume s'inscrivent dans des perspectives méthodologiques et théoriques différentes, tous partagent un même souci d'appuyer leurs leurs analyses, de manière directe ou indirecte, sur des données attestées.

Marie-Hélène Côté se propose tout d'abord d'explorer la question de la variation diatopique dans la réalisation de la liaison, un aspect qui, contrairement au schwa par exemple, a été très peu traité. Elle y propose une nouvelle typologie de la liaison basée sur la présence/absence de liaison variable et de liaisons non standard. Cette typologie permet de distinguer quatre grands groupes qui correspondent à l'Afrique, au Canada, à la Louisiane et à l'Europe.

Giulia Barreca et George Christodoulides proposent ensuite une analyse de la liaison variable en s'appuyant sur une partie de la base PFC qu'ils ont annotée morpho-syntaxiquement. Ils montrent notamment que la grande majorité des liaisons variables est limitée à un nombre restreint de constructions très productives. Ils montrent également, à travers une analyse détaillée du comportement des adverbes, qu'une approche constructionnelle basée sur les catégories grammaticales n'est pas suffisante, et qu'il est nécessaire de prendre en considération les liens de dépendance syntaxique qui affectent le mot liaisonnant.

Deux études viennent ensuite éclairer la diachronie récente du phénomène. Olivier Baude et Céline Dugua entreprennent une étude longitudinale des corpus d'Orléans (ESLO I et ESLO2, construits à 40 ans d'intervalle), de manière à rendre compte de la diachronie (en temps réel) de la liaison chez des locuteurs qui ne sont pas des professionnels de la parole publique. Leurs résultats mettent en lumière le fait que la liaison variable est globalement stable, avec néanmoins une forte variation inter-individuelle dans les deux corpus. Bernard Laks et Julie Peuvergne explorent quant à eux la réalisation de la liaison dans un corpus récent de discours politiques, résultats qu'ils confrontent aux travaux antérieurs sur cette population de professionnels de la parole publique.

Les deux études suivantes apportent un nouveau regard sur l'acquisition de la liaison: Céline Dugua, Aurélie Nardy, Loïc Liégeois, Jean-Pierre Chevrot et Damien Chabanal se proposent d'éprouver le modèle développemental de l'acquisition de la liaison de Chevrot, Dugua et Fayol (2009), en comparant la liaison en contexte prénominal et préverbal. Ils montrent notamment que les erreurs de segmentation qui consistent à rattacher la CL au Mot2, bien qu'elles soient fréquentes en contexte prénominal (ex : zours, navion), sont absentes en contexte préverbal. Isabelle Racine et Sylvain Detey abordent quant à eux la question de l'acquisition chez les apprenants non natifs. Ils mettent en exergue les difficultés spécifiques, en particulier en production, que pose la liaison aux apprenants du français langue étrangère. Ils insistent sur la nécessité d'intégrer dans les ressources pédagogiques un travail explicite portant sur la 


\section{Julien Eychenne et Bernard Laks}

nature de la consonne de liaison, son rapport à la graphie, et l'enchaînement syllabique.

Enfin, Elissa Pustka, Marc Chalier et Luise Jansen se proposent de réévaluer la notion de norme de prononciation de la liaison dans une perspective panfrancophone, sur la base d'un corpus de parole télévisée.

Pris dans son ensemble, ce numéro offre ainsi un panorama représentatif des questions empiriques, descriptives et théoriques que soulève la liaison à l'heure actuelle.

\section{ACKNOWLEDGEMENTS}

La contribution de Julien Eychenne à cet article a été soutenu par le Hankuk University of Foreign Studies Research Fund 2016.

Adresse pour correspondance:

e-mail: jeychenne@hufs.ac.kr

\section{RÉFÉRENCES}

Barreca, G. (20I 5). L'acquisition de la liaison chez des apprenants italophones. Des atouts d'un corpus de natifs pour l'étude de la liaison en français langue étrangère (FLE). Thèse de doctorat non publiée, Université de Paris Ouest Nanterre La Défense.

Bonami, O. et Boyé, G. (2003). Supplétion et classes flexionnelles dans la conjugaison du français. Langages, I 52: I02-I26.

Bonami, O., G. Boyé et J. Tseng (2005). Sur la grammaire des consonnes latentes. Langages, I 58: 89-100.

Bybee, J. (200Ia). Frequency effects on French Liaison. In: J. Bybee et P. Hopper (dir.), Frequency and the Emergence of Linguistic Structure. Amsterdam: John Benjamins, pp. 337-359.

Bybee, J. (200Ib). Phonology and Language Use. Cambridge: Cambridge University Press.

Côté, M.-H. (2005). Le statut lexical des consonnes de liaison. Langages, I 58: 66-78.

Côté, M.-H. (201 I). French Liaison. In: M. van Oostendorp, C. Ewen, E. Hume et K. Rice (dir.), The Blackwell Companion to Phonology. Malden: WileyBlackwell, pp. 2685-27Iо.

Côté, M.-H. (2OI4). Liaison et assibilation en français laurentien. In: Soum et al. (20I4).

Damourette, J. et Pichon, E. (I9I I-I927). Des mots à la pensée. Essai de grammaire de la langue française. Paris: D’Artrey.

Delattre, P. (195I). Principes de phonétique française à l'usage des étudiants anglo-américains. Middleburry: Middleburry College.

Delattre, P. (1966). Studies in French and Comparative Phonetics. Londres: Mouton.

Dell, F. (I973). Les règles et les sons: introduction à la phonologie générative. Paris: Hermann.

Durand, J., Laks, B. Calderone, B. et A. Tchobanov (20I I). Que savons-nous de la liaison aujourd'hui? Langue Française, I69: I03-35.

Durand, J., et Lyche, C. (2008). French liaison in the light of corpus data. Journal of French Language Studies, I8(I): 33-66.

Durand, M. (1936). Le genre grammatical en français parlé à Paris et dans la région parisienne. Paris: Bibliothèque du Français Moderne. 


\section{La liaison en français contemporain}

Encrevé, P. (1988). La liaison avec et sans enchaînements: phonologie tridimentionnelle et usages $d u$ français. Paris: Le Seuil.

Fouché, P. (1959). Traité de prononciation française, Paris: Klincksieck.

Goldsmith, J. et Laks, B. (dir.) (2000). On the history of phonology. Numéro spécial de Folia Linguistica 34 .

Gougenheim, G. (1938). Le système grammatical de la langue française. Paris: D’Artrey et Rondeix-D'Artrey.

Grammont, M. (I9I4). Traité pratique de prononciation française. Paris: Delagrave.

Grevisse, M. (1936). Le bon usage. Paris: Gembloux.

Hindret, J. (I687). L'Art de bien prononcer et de bien parler la langue françoise. Dedié à monseigneur le duc de Bourgogne. Par le sieur J.H. Paris: Laurent d'Houry.

Labov, W. (1994). Principles of Linguistic Change: Internal Factors. Oxford: Blackwell.

Laks, B. (2005). La liaison et l'illusion. Langages, I 58: IOI-I26.

Laks, B. et Calderone, B. (2013). French liaison and the lexical repository. In: C. Celata et S. Calamai (dir.), Advances in Sociophonetics. Amsterdam: Benjamins.

Martinon, $\mathrm{P}$. (I9I3). Comment on prononce le français: traité complet de prononciation pratique avec les noms propres et les mots étrangers. Paris: Larousse.

Milner, J.-C. (I967). French truncation rule. Quaterly Progress Report of the Research Laboratory of Electronics, MIT 86.

Morin, Y.-C. (I986). On the morphologization of word-final consonant deletion in French. In: H. Andersen (dir.), Sandhi Phenomena in the languages of Europe. Berlin: Mouton de Gruyter, pp. I67-2 Io.

Morin, Y.-C. (2003). Remarks on prenominal liaison consonants in French. In: S. Ploch (dir.), Living on the Edge. 28 papers in Honour of Jonathan Kaye. Berlin: Mouton de Gruyter, pp. 385-400.

Morin, Y.-C. (2005a). La liaison relève-t-elle d'une tendance à éviter les hiatus? Réflexions sur son évolution historique. Langages, I 58: 8-23.

Morin, Y.-C. (2005b). Liaison et enchaînement dans les vers aux XVIe et XVIIe siècles. In: J.-M. Gouvard (dir.), De la langue au style. Lyon: Presses Universitaires de Lyon, pp. 299-3 I 8.

Morin, Y.-C. et Kaye, J. (1982). The syntactic bases for French liaison. Journal of Linguistics, I 8: 29I-330.

Pichon, E. (1938). Genre et questions connexes (sur les pas de Mlle Durand). Le Français Moderne, 6: I07-I26.

Schane, S. (1965). The phonological and morphological structure of French. Thèse de doctorat, MIT.

Schane, S. (1967). L'élision et la liaison en français. Langages, 8: 37-59.

Schane, S. (I974). There is no French truncation rule. In: J. Campbell, M. Goldin, et M. C. Wang (dir.), Linguistic Studies in Romance Languages. Washington: Georgetown University Press, pp. 89-99.

Scheer, T. (20 I 5). Précis de structure syllabique. Accompagné d'un apparat critique. Lyon: ENS Editions.

Scheer, T., Wauquier, S. et Encrevé, P. (2015). Autosegmental news from $h$ aspiré and liaison without enchaînement. Communication aux 1zèmes Rencontres du Réseau Français de Phonologie (RFP), Bordeaux.

Selkirk, E. O. (1972). The Phrase Phonology of English and French. Cambridge MA: MIT. Soum, C., A. Coquillon et J.-P. Chevrot (dir.) (2OI4). La liaison: approches contemporaines. Berne: Peter Lang. 


\section{Julien Eychenne et Bernard Laks}

Tranel, B. (1981). Concreteness in Generative Phonology. Evidence from French. Berkeley: University of California Press.

Tranel, B. (I995). Current Issues in French Phonology: Liaison and Position Theories. In: J. Goldsmith (dir.), The Handbook of Phonological Theory. Oxford: Blackwell, pp. 798-8 г6.

Tranel, B. (2000). Aspects de la phonologie du français et la théorie de l'optimalité. Langue française, I26: 39-72. 